\title{
Factores asociados a sintomatología clínica de miedo y ansiedad en pacientes atendidos en Odontología
}

\author{
Katherine Arrieta Vergara ${ }^{a}$, Shyrley Díaz Cárdenas ${ }^{\mathrm{b}}$, Johana Verbel Bohórquez ${ }^{\mathrm{c}}$,
} Nathalie Hawasly Pastrana ${ }^{c}$

\begin{abstract}
a Odontóloga, Magister en Salud Pública y especialista en Gerencia en Salud. Facultad de Odontología. Universidad de Cartagena de Indias (Colombia). b Odontóloga, Magister en Salud Pública y especialista en Gerencia en Salud y Salud Familiar. Facultad de Odontología. Universidad de Cartagena de Indias (Colombia). c Odontóloga. Facultad de Odontología. Universidad de Cartagena de Indias (Colombia).

Correspondencia: Katherine Arrieta Vergara. Facultad de Odontología, Departamento de Odontología Preventiva y Social. Universidad de Cartagena, Campus de la Salud, Barrio Zaragocilla. Cartagena de Indias (Colombia). Correo electrónico: katherinearrieta@gmail.com
\end{abstract}

Recibido el 25 de abril de 2013. Aceptado para su publicación el 28 de mayo de 2013.

\begin{abstract}
RESUMEN
Objetivo: Estimar el grado de asociación entre factores asociados a sintomatología clínica de miedo y ansiedad dental en pacientes adultos de la Facultad de Odontología de la Universidad de Cartagena de Indias (Colombia).

Diseño del estudio: Estudio observacional transversal.

Emplazamiento: Facultad de Odontología de la Universidad de Cartagena de Indias (Colombia). Participantes: 460 pacientes adultos seleccionados por muestreo aleatorio simple.

Mediciones principales: Se utilizó un instrumento que incluyó factores socio-demográficos y conductuales, y los cuestionarios de cribado: Escala de Goldberg, Kleinknecht's Dental Fear Survey (DFS) y Short Version Dental Anxiety Scale (SDAI). Los datos fueron analizados a partir de proporciones, asumiendo intervalos de confianza del 95\%, razones de disparidad (Odds ratio) y regresión logística, utilizando el programa STATA versión $10.0^{\circledR}$.

Resultados: La prevalencia de sintomatología clínica de miedo y ansiedad dental fue $77,1 \%$ (IC 95\% 73,3-81) y $62,8 \%$ (IC 95\% 58,3-67,2) respectivamente. Se presentó asociación estadísticamente significativa del miedo dental con el sexo femenino (OR=1,9; IC95\% 1,2 - 2,9) y la experiencia traumática previa en la consulta odontológica (OR=3,2; IC95\% 1,73-6,28) y de la ansiedad en la consulta odontológica con el sexo femenino (OR=2; IC95\% 1,3-2,9), la experiencia traumática previa en la consulta odontológica $(\mathrm{OR}=2,2$; IC95\% 1,4-3,6), influencia negativa de los familiares $(\mathrm{OR}=1,5$; IC95\% 1,07-2,2), percepción del paciente acerca de su salud oral (OR=1,5; IC95\% 1,04-2,2), y el miedo en la consulta odontológica (OR=39,8 IC95\% 19,2- 82,5).

Conclusiones: La prevalencia de miedo y ansiedad dental en los sujetos fue alta; el sexo femenino, la influencia negativa de familiares y las experiencias traumáticas previas en la consulta odontológica son factores de riesgo para la aparición de miedo y ansiedad en la consulta odontológica.
\end{abstract}

PALABRAS CLAVE: Ansiedad al Tratamiento Odontológico. Miedo. Odontología.

\section{ABSTRACT}

Objective: Estimate the degree of association between factors associated with clinical symptomatology of fear and anxiety in adult patients at the Faculty of Dentistry of the university of Cartagena de Indias (Colombia).

Design: Cross sectional observational study.

Location: Faculty of Dentistry of the university of Cartagena de Indias (Colombia).

Participants: 460 adult patients selected by simple random sampling.

Main Surveyed Data: An instrument which included socio-demographic and behavioural factors was used and a screening questionnaire: Goldberg scale, Kleinknecht's Dental Fear Survey (DFS) and Short Version Dental Anxiety Scale (SDAI). Data were analysed based on proportions, assuming a 95\% confidence interval, odds ratio and logistic regression, using the STATA programme version $10.0^{\circledR}$.

Results: The prevalence of clinical symptomatology of fear and dental anxiety

was $77.1 \%$ (IC 95\% 73.3-81) and $62.8 \%$ (IC 95\% 58.3-67.2) respectively. Statistically significant dental fear associated with the female sex was recorded (OR=1.9; IC95\% 1.2-2.9) and previous traumatic experience in the dental surgery (OR=3.2; IC95\% 1.73-6.28) and anxiety in the dental surgery with the female sex (OR=2; IC95\% 1.3-2.9), previous traumatic experience in the dental surgery $(\mathrm{OR}=2.2$; IC95\% 1.4-3.6), negative influence from members of the family $(\mathrm{OR}=1.5$; IC95\% 1.07-2.2), patient's perception of dental health (OR=1.5; IC95\% 1.04-2.2), and fear in the dental surgery (OR=39.8 IC95\% 19.2- 82.5).

Conclusions: Prevalence of dental fear and anxiety in the subjects was high; the female sex, negative influence from members of the family and previous traumatic experiences in the dental surgery are risk factors causing fear and anxiety in the dental surgery

KEYWORDS: Dental treatment anxiety. Fear. Dentistry. 


\section{INTRODUCCIÓN}

Alrededor de la consulta odontológica se generan en el paciente emociones como el miedo y la ansiedad, que pueden influir directamente en el diseño de un plan de tratamiento y ejecución de un procedimiento ${ }^{1,2}$. El miedo y la ansiedad dental pueden surgir por múltiples causas: fobia a las agujas, extracciones dentales, sonido de la pieza de mano de alta velocidad, percepción negativa del odontólogo frente a la salud oral del paciente, entre otros $^{3,4}$. Se estima que entre el $10 \%$ y el $15 \%$ de la población mundial experimenta ansiedad al acudir al odontólogo, lo que puede inducir a cancelar o posponer su cita, teniendo efectos negativos sobre su salud oral5, ${ }^{5}$.

La ansiedad y los miedos dentales, desde la perspectiva conductual, se consideran patrones multidimensionales y aprendidos de comportamiento que resultan desadaptativos con relación al tratamiento odontológico ${ }^{5}$. Tradicionalmente, los términos miedo y ansiedad se diferencian porque el primero es una reacción emocional inmediata ante un peligro actual e identificable, mientras que la segunda es un estado emocional más difuso y sin una fuente externa de amenaza reconocible ${ }^{7}$, es decir, no siempre varía en proporción directa al peligro real (objetivo) de la situación?.

Berggren $^{9}$ afirma que la ansiedad dental crea su propio círculo vicioso, en el que la evasión del tratamiento dental logra un deterioro de la dentición y origina sentimientos de culpa e inferioridad en el paciente. Este "conflicto social" resulta en una nueva evasión, que conllevará al detrimento de la salud oral del individuo a partir de la pérdida de órganos dentarios, así como de la inconformidad estética y funcional, determinando el estilo de vida de la persona ${ }^{1,10}$ y comprometiendo su bienestar biopsicosocial.

Es poco lo que se conoce sobre esta temática a nivel de la Ciudad de Cartagena de Indias (Colombia); por tanto, la finalidad de este estudio es obtener evidencia científica sobre la presencia de sintomatología clínica de miedo y ansiedad y su grado de asociación con los factores asociados en pacientes adultos que acuden a la Facultad de Odontología de la Universidad de Cartagena (Colombia).

\section{SUJETOS Y MÉTODOS}

Estudio de corte transversal realizado en pacientes adultos atendidos en las clínicas de la Facultad de Odontología de la Universidad de Cartagena (Colombia) durante el primer semestre del año 2012. Para el cálculo del tamaño de la muestra se tuvo en cuenta una población universo de 795 pacientes, una confianza del $95 \%$, un error del $2 \%$ y una frecuencia esperada del fenómeno objeto de estudio del 30\% (prevalencia de ansiedad en la consulta odontológica) ${ }^{11}$, obteniéndose una muestra de 460 pacientes, los cuales fueron seleccionados a través de un muestreo aleatorio simple con reemplazo. Para tal fin se utilizó una lista de números aleatorios con la cual fueron escogidos los individuos de la lista de pacientes atendidos en las clínicas odontológicas durante el periodo de estudio; si en el momento de la recolección de la información el paciente seleccionado no se encontraba en la clínica, era reemplazado por otro; para la participación de los sujetos su tuvieron en cuenta los siguientes criterios: tener historia clínica vigente, y firmar consentimiento informado teniendo en cuenta las disposiciones internacionales: Declaración de Helsinki (modificación de Edimburgo 2000) y la normativa para Colombia (resolución 8430 de 1993 Ministerio de Salud). Se excluyeron personas con deficiencias mentales no aptas para la toma de decisiones.

Para la recolección de la información se diseñaron dos instrumentos. El primero, de tipo cuestionario anónimo y de autorreporte con 53 preguntas, previamente evaluado con una prueba piloto, incluyéndose la comprensión de las preguntas, semántica, sintaxis, coherencia y pertinencia; este cuestionario se administró en una primera etapa e incluyó la siguiente información: factores socio-demográficos (edad: adulto joven [2044 años], adulto medio [45-59 años], adulto mayor [mayor a 60 años], sexo (femenino, masculino), y escolaridad (analfabeta, primaria incompleta y completa, secundaria incompleta y completa, formación técnica profesional, nivel tecnólogo y profesional universitario), presencia de enfermedades crónicas y sistémicas, así como factores conductuales (relacionados con la consulta [experiencia traumática en la consulta odontológica, percepción de salud oral] y factores familiares [influencias familiares negativas sobre la atención odontológica]). Se incluyeron los siguientes test de cribado:

1. Escala de Goldberg ${ }^{12}$ : escala para la detección de trastornos de ansiedad, utilizada por médicos no psiquiatras como instrumento de cribaje para los trastornos de ansiedad y depresión sin precisar estandarización. La versión en castellano ha sido validada por Montón C y cols. (1993) ${ }^{13}$ y consta de dos escalas, una de ansiedad y otra de depresión, con 9 ítems cada una, todos ellos de respuesta dicotómica (sí / no); se da una puntuación independiente para cada escala, con un punto 
para cada respuesta afirmativa; se interroga al paciente sobre si ha presentado en las últimas dos semanas alguno de los síntomas a los que hacen referencia los ítems; no se puntúan los síntomas que duren menos de dos semanas o que sean de leve intensidad. La escala de ansiedad se estructura en 4 ítems iniciales de despistaje para determinar si es o no probable que exista un trastorno mental, y un segundo grupo de 5 ítems que se formulan sólo si se obtienen respuestas positivas (2 o más respuestas en los 4 primeros ítems) a las preguntas de despistaje y su punto de corte corresponde $\geq 4$ en total para la presencia de ansiedad.

2. Kleinknecht Dental Fear Survey (DFS) ${ }^{14}$ : escala establecida para identificar estímulos y reacciones específicas del miedo dental, útil en adultos, que consiste en veinte ítems relacionados con la evitación de citas con el dentista, reacciones físicas tenidas durante el trabajo dental y miedo suscitado por diversos aspectos de la situación dental. Es calificada por el cálculo de la suma de los 20 ítems. Los resultados pueden variar desde 20 a 100. Una puntuación total de 20 indica "sin miedo", una puntuación de 21-40 significa "miedo bajo", un resultado de 41-79 muestra "miedo moderado", mientras que una puntuación de 80 hasta 100 indicaría "miedo alto"

3. Short Versión Dental Anxiety Scale (SDAl) ${ }^{1}$ : instrumento de autorreporte que consta de 9 ítems que evalúan las situaciones relativas al tratamiento odontológico en que el paciente presenta ansiedad. Cada ítem es calificado en una escala likert de 5 puntos que evalúa frecuencia de presentación de cada situación. Fue desarrollado con base en el análisis del Inventario de Ansiedad Dental; este inventario ha mostrado buenos niveles de confiabilidad y validez y altas correlaciones con inventarios de ansiedad dental como el DAS ${ }^{16}$. Dada la carencia de instrumentos validados para la población colombiana, en el estudio de Caycedo et al $^{1}$ se validó la escala SDAI arrojando puntaje de confiabilidad alfa de Cronbach de 0,957. Esta es calificada por el cálculo de la suma de los nueve ítems. Las puntuaciones más altas reflejan la ansiedad extrema. Una puntuación en el rango 0-13 indica "sin ansiedad", una puntuación de 1420 significa "levemente ansioso", un resultado de 21-29 indica " moderadamente ansioso", mientras que una puntuación de 30-45 muestra "extremadamente ansioso"1.

En una segunda etapa, se realizaron los exámenes clínicos para obtener el diagnóstico del estado de salud bucal de los sujetos, mediante un segundo instrumento, que incluyó el índice COP-D Klein y Palmer $^{17}$ (que evalúa la prevalencia de caries, a la vez que señala la experiencia de caries pasada y presente; se obtiene de la sumatoria de los dientes permanentes cariados, obturados y perdidos [incluyendo las extracciones indicadas] y considerando solo 28 dientes), e indagaba por la presencia de restos radiculares, enfermedad periodontal y prótesis dentales. Para obtener la validez de las mediciones se realizaron dos sesiones de estandarización con una diferencia de tiempo entre las sesiones de una semana, en un total de 20 pacientes, usando como referencia a un examinador experto en caries (estándar de oro), teniendo como valor para la selección un Kappa Cohen de 0,80 a nivel intraexaminador y 0,75 interexaminador. Tras este procedimiento se obtuvieron dos examinadores estandarizados: para el examinador 1 el valor intraexaminador (concordancia en las mediciones del mismo examinador durante las sesiones de diagnóstico) fue de 0,84 y el valor interexaminador (concordancia del examinador con el estándar de oro) de 0,80 ; y para el examinador 2 los valores fueron de 0,81 y 0,78 respectivamente. Todas las evaluaciones fueron hechas en las clínicas de la Facultad de Odontología de la institución universitaria, utilizando luz artificial de la unidad odontológica, espejo bucal número cinco y sonda periodontal.

La información fue organizada y depurada a través de una base de datos en Microsoft Excel ${ }^{\circledR}$ 2010, luego fue analizada e interpretada en el programa estadístico STATA $10.0^{\circledR}$, versión para Windows ${ }^{\circledR}$ 10.0. Los datos se analizaron a través de distribuciones de frecuencia y proporciones, asumiendo intervalos de confianza del 95\%. Se realizó análisis inferencial usando la prueba chi ${ }^{2}$ para las relaciones entre el miedo y la ansiedad y los factores asociados, asumiendo un límite de 0,05 para la significación. La fuerza de asociación se estimó mediante Razones de Odds con intervalos de confianza al 95\%. Además, para el análisis multivariante se utilizó la regresión logística nominal, teniendo en cuenta las variables que marcaron una probabilidad igual o menor al 5\%.

\section{RESULTADOS}

La edad media de los participantes fue 42,6 años (DE=15,7). El $63,4 \%$ fueron mujeres, el $25,8 \%$ terminaron sus estudios de secundaria y el estrato socioeconómico con mayor porcentaje fue estrato bajo (tabla 1). El 39,5\% (IC95\% 35$44 \%$ ) presentaron resultados positivos (escala de Goldberg) para posible presencia de sintomatología ansiosa de importancia clínica; con respecto a la ansiedad en la consulta odontológica (SDAI) se encontró una prevalencia de 62,8\% (IC95\% 58,3$67,2 \%$ ) y una prevalencia de miedo dental (DFS) del 


\begin{tabular}{|c|c|c|}
\hline Variable & Frecuencia $(n=460)$ & Porcentaje (\%) \\
\hline \multicolumn{3}{|l|}{ Sexo } \\
\hline Femenino & 292 & 63,4 \\
\hline Masculino & 168 & 36,5 \\
\hline \multicolumn{3}{|l|}{ Estado civil } \\
\hline Soltero & 151 & 32,8 \\
\hline Casado & 193 & 41,9 \\
\hline Divorciado & 15 & 3,2 \\
\hline Viudo & 14 & 3,0 \\
\hline Unión libre & 75 & 16,3 \\
\hline Separado & 12 & 2,6 \\
\hline \multicolumn{3}{|l|}{ Procedencia } \\
\hline Urbana & 319 & 69,3 \\
\hline Rural & 141 & 30,6 \\
\hline \multicolumn{3}{|l|}{ Escolaridad } \\
\hline Analfabetos & 8 & 1,7 \\
\hline Primaria incompleta & 37 & 8,0 \\
\hline Primaria completa & 45 & 9,7 \\
\hline Secundaria incompleta & 67 & 14,5 \\
\hline Secundaria completa & 119 & 25,8 \\
\hline Formación técnica profesional (2 años de estudios) & 77 & 16,7 \\
\hline Nivel tecnológico (3 años de estudio) & 22 & 4,7 \\
\hline Profesional universitario (4-5 años de estudio) & 85 & 18,4 \\
\hline
\end{tabular}

Tabla 1. Variables socio-demográficas de los pacientes adultos atendidos en las clínicas de la Facultad de Odontología de la Universidad de Cartagena (Colombia).

$77,1 \%$ (IC95\% 73,3-81\%) (tabla 2). Se obtuvo una consistencia interna Alfa de Cronbach de 0,84 para los trastornos de Ansiedad (escala Goldberg), 0,75 para ansiedad en la consulta odontológica (SDAl) y 0,88 para el miedo dental (DFS).

En salud oral, el índice COP-D fue de 13 (DE=7): Cariados: 2,5 ( $\mathrm{DE}=2,2)$, Obturados: 3,4 ( $\mathrm{DE}=2,6)$, Perdidos: 7 ( $\mathrm{DE}=7,9$ ); el estado de higiene oral fue deficiente (placa bacteriana de $20 \%$ al $100 \%$ ) para el $96 \%$ de los pacientes.

En la tabla 3 se observan las relaciones con significación estadística encontradas en el presente estudio para los síntomas de miedo dental. Estas se modelaron por regresión logística, y el mejor modelo surgió con dos variables: sexo (femenino) y experiencia traumática previa en la consulta odontológica $\left(p=0,000 ; \chi^{2}=23,8\right)$. En la tabla 4 se observan las relaciones con significación estadística encontradas en el presente estudio para los síntomas de ansiedad en la consulta odontológica. Al realizar el análisis de regresión logística los factores que mejor explicaron la presencia de sintomatología ansiosa en los pacientes de odontología fueron sexo (femenino) y el miedo dental $\left(p=0,000 ; \chi^{2}=184,3\right)$.

\section{DISCUSIÓN}

Según los resultados obtenidos en esta investigación, la prevalencia de miedo y ansiedad dental en las clínicas odontológicas se considera importante, ya que más de la mitad de los sujetos de estudio la manifestaron. Estos resultados superan a los observados por otros autores ${ }^{11,18,19}$. Estas diferencias pueden obedecer al bajo estrato socioeconómico al que pertenecen los sujetos de estudios, el cual ha sido asociado en la literatura a la presencia de trastornos mentales ${ }^{20}$; es así que el no disponer de recursos económicos hace que la atención en salud oral se limite a la atención de 


\begin{tabular}{|c|c|c|c|}
\hline Variable & $\begin{array}{l}\text { Frecuencia } \\
\qquad(n=460)\end{array}$ & Porcentaje (\%) & $\begin{array}{c}\text { Intervalo de } \\
\text { confianza del } 95 \%\end{array}$ \\
\hline \multicolumn{4}{|c|}{ Trastorno de ansiedad (Goldberg) } \\
\hline Sí & 182 & 39,5 & $35,0-44,0$ \\
\hline No & 278 & 60,4 & $55,9-64,9$ \\
\hline $\begin{array}{l}\text { Ansiedad en la } \\
\text { odontológica (SDAI) }\end{array}$ & & & \\
\hline No & 171 & 37,1 & $32,7-41,6$ \\
\hline Sí & 289 & 62,8 & $58,3-67,2$ \\
\hline \multicolumn{4}{|c|}{$\begin{array}{l}\text { Niveles de ansiedad en la consulta } \\
\text { odontológica }\end{array}$} \\
\hline Sin ansiedad & 171 & 37,1 & $32,7-41,6$ \\
\hline Levemente ansioso & 166 & 36,0 & $31,6-40,4$ \\
\hline Moderadamente ansioso & 100 & 21,7 & $17,9-25,5$ \\
\hline Extremadamente ansioso & 23 & 5,0 & $3,0-6,0$ \\
\hline \multicolumn{4}{|l|}{ Miedo dental (DFS) } \\
\hline No & 106 & 23,0 & $19,1-26,9$ \\
\hline Sí & 355 & 77,1 & $73,3-81,0$ \\
\hline \multicolumn{4}{|l|}{ Niveles de miedo dental } \\
\hline Ausente & 106 & 23,0 & $19,1-26,9$ \\
\hline Bajo & 281 & 61,0 & $56,6-65,5$ \\
\hline Moderado & 73 & 15,8 & $12,5-19,2$ \\
\hline
\end{tabular}

Tabla 2. Prevalencia de trastornos de ansiedad, ansiedad en la consulta odontológica y miedo dental en pacientes adultos atendidos en las clínicas de la Facultad de Odontología de la Universidad de Cartagena (Colombia).

la morbilidad y no de la prevención, requiriendo de tratamientos más costoso, invasivos o dolorosos. Otra posible explicación de las diferencias está en el gran porcentaje de mujeres que respondieron la encuesta, quienes presentaron más casos de ansiedad y miedo dental.

Con respecto al sexo, las mujeres presentaron más ansiedad y miedo en la consulta odontológica que los hombres; esta diferencia fue estadísticamente significativa, similar a estudios previos realizados por Caycedo et $a^{1}{ }^{1}$. y Álvarez y Casanova ${ }^{7}$; dentro de los factores que podrían explicar las diferencias están los factores psicosociales y socioculturales que incluyen las diferencias en el tipo de afrontamiento de la persona, rol sexual, pobreza, nivel educativo, apoyo social, adversidades durante la infancia, normas culturales situaciones estresantes, comorbilidad, rasgos de personalidad, predisposición genética, u hormonas sexuales entre otros ${ }^{1,7,21,22}$.

Se encontró que haber tenido una experiencia traumática en la consulta odontológica es un factor de riesgo para la aparición de miedo dental y ansiedad en la consulta odontológica; una de las explicaciones más tradicionales para la adquisición de ansiedad y miedo dental se deriva del modelo de condicionamiento clásico, el cual explica que el miedo es adquirido tras una experiencia negativa o traumática ${ }^{23}$; Locker, Shapiro y Liddell ${ }^{24}$ reportan que la ansiedad dental se relaciona específicamente con un tratamiento invasivo o doloroso, evocando un deseo para evitar situaciones dentales posteriores.

La relación de influencia negativa por parte de familiares, amigos o conocidos se identificó con factor de riesgo de sintomatología clínica de ansiedad en la consulta odontológica, similar a los resultados de Carillo et al, ${ }^{25}$ que afirman que los niveles de ansiedad dental en los pacientes están influenciados o conectados por sus familiares; este hallazgo ha sido propuesto como justificación de un mecanismo modelado por contagio emocional de miedo dental entre los miembros familiares. De 


\begin{tabular}{|c|c|c|c|c|c|c|}
\hline \multirow{3}{*}{ Variables } & \multicolumn{6}{|c|}{ Miedo dental } \\
\hline & \multicolumn{4}{|c|}{ Bivariado } & \multicolumn{2}{|c|}{ Multivariado } \\
\hline & $\begin{array}{c}\text { Sí } \\
\text { n (\%) }\end{array}$ & $\begin{array}{c}\text { No } \\
\text { n (\%) }\end{array}$ & OR & $\mathrm{IC} 95 \%$ & OR & IC95\% \\
\hline \multicolumn{7}{|l|}{ Sexo } \\
\hline Femenino & $238(81,5)$ & $54(18,4)$ & $1,9^{*}$ & $1,2-2,9$ & $1,9^{* *}$ & $1,2-2,9$ \\
\hline Masculino (ref) & $117(69,6)$ & $51(30,3)$ & 1,0 & & & \\
\hline \multicolumn{7}{|l|}{ Edad } \\
\hline Adulto mayor / maduro & $169(74,1)$ & $59(25,8)$ & 0,72 & $0,40-1,09$ & & \\
\hline Adulto joven (ref) & $186(80,1)$ & $46(10,2)$ & 1,0 & & & \\
\hline \multicolumn{7}{|l|}{ Estado civil } \\
\hline Soltero & $152(79,1)$ & $40(20,8)$ & 1,2 & $0,7-1,9$ & & \\
\hline Casado (ref) & $203(75,7)$ & $65(24,2)$ & 1,0 & & & \\
\hline \multicolumn{7}{|l|}{ Enfermedad } \\
\hline Sí & $169(76,8)$ & $51(23,1)$ & 0,8 & $0,9-2,0$ & & \\
\hline No (ref) & $186(77,5)$ & $54(22,5)$ & 1,0 & & & \\
\hline \multicolumn{7}{|c|}{ Experiencia previa traumática } \\
\hline Sí & $105(89,7)$ & $12(10,2)$ & $3,2^{\star \star \star}$ & $1,73-6,28$ & $3,2^{* * *}$ & $1,6-6,1$ \\
\hline No (ref) & $250(72,8)$ & $93(27,1)$ & & & & \\
\hline \multicolumn{7}{|l|}{ Influencia negativa } \\
\hline Sí & $194(79,1)$ & $51(20,8)$ & 1,2 & $0,8-1,9$ & & \\
\hline No (ref) & $161(74,8)$ & $54(25,1)$ & 1,0 & & & \\
\hline \multicolumn{7}{|l|}{ Víctima de equivocación } \\
\hline Sí & $62(72,9)$ & $23(27,06)$ & 0,8 & $0,5-1,2$ & & \\
\hline No (ref) & $293(78,1)$ & $82(21,8)$ & 1,0 & & & \\
\hline \multicolumn{7}{|l|}{ Percepción salud oral } \\
\hline Buena & $208(75)$ & $69(25)$ & 0,8 & $0,5-1,2$ & & \\
\hline Mala (ref) & $147(80,3)$ & $36(19,6)$ & 1,0 & & & \\
\hline \multicolumn{7}{|l|}{ Porcentaje de placa } \\
\hline $21-100 \%$ & $338(76,4)$ & $104(23,3)$ & 5,2 & $0,6-39,7$ & & \\
\hline $0-20 \%$ & $17(94,4)$ & $1(5,5)$ & 1,0 & & & \\
\hline \multicolumn{7}{|l|}{ Gingivitis } \\
\hline Presencia & $329(76,6)$ & $100(23,3)$ & 0,6 & $0,2-1,6$ & & \\
\hline Ausencia (ref) & $26(83,8)$ & $5(16,1)$ & 1,0 & & & \\
\hline \multicolumn{7}{|l|}{ Periodontitis } \\
\hline Presencia & $150(76,1)$ & $47(23,8)$ & 0,8 & $0,5-1,3$ & & \\
\hline Ausencia (ref) & $205(78)$ & $58(22)$ & 1,0 & & & \\
\hline \multicolumn{7}{|c|}{ Trastorno de ansiedad (Goldberg) } \\
\hline Con ansiedad & $146(80,2)$ & $36(19,7)$ & 1,3 & $0,8-2,1$ & & \\
\hline Sin ansiedad (ref) & $209(75,1)$ & $69(24,8)$ & 1,0 & & & \\
\hline
\end{tabular}

Tabla 3. Asociación entre el miedo dental en la consulta odontológica con variables socio-demográficas, experiencia previa, percepción y salud oral de individuos atendidos en las clínicas de la Facultad de Odontología de la Universidad de Cartagena (Colombia).

Significación del modelo OR $\left(p=0,000 ; \chi^{2}=23,8\right){ }^{*} p=0,004{ }^{* *} p=0,005^{* * *} p=0,000$ 


\begin{tabular}{|c|c|c|c|c|c|c|}
\hline \multirow{3}{*}{ Variables } & \multicolumn{6}{|c|}{ Ansiedad en odontología } \\
\hline & \multicolumn{4}{|c|}{ Bivariado } & \multicolumn{2}{|c|}{ Multivariado } \\
\hline & $\begin{array}{c}\text { Sí } \\
\text { n (\%) }\end{array}$ & $\begin{array}{c}\text { No } \\
\text { n (\%) }\end{array}$ & OR & IC95\% & OR & IC95\% \\
\hline \multicolumn{7}{|l|}{ Sexo } \\
\hline Femenino & $201(68,8)$ & $91(31,1)$ & $2,0^{*}$ & \multirow{2}{*}{$1,3-2,9$} & \multirow{2}{*}{$1,73^{* *}$} & \multirow{2}{*}{$1,06-2,8$} \\
\hline Masculino (ref) & $88(52,3)$ & $80(47,6)$ & 1,0 & & & \\
\hline \multicolumn{7}{|l|}{ Edad } \\
\hline Adulto mayor / maduro & $135(68,8)$ & $93(40,7)$ & 0,73 & \multirow{2}{*}{$0,50-1,07$} & & \\
\hline Adulto joven (ref) & $154(66,3)$ & $78(33,6)$ & 1,0 & & & \\
\hline \multicolumn{7}{|l|}{ Estado civil } \\
\hline Soltero & $124(64,5)$ & $68(35,4)$ & 1,1 & \multirow{2}{*}{$0,7-1,6$} & & \\
\hline Casado (ref) & $165(61,5)$ & $103(38,4)$ & 1,0 & & & \\
\hline \multicolumn{7}{|l|}{ Enfermedad } \\
\hline Sí & $147(66,8)$ & $73(33,1)$ & 1,3 & \multirow{2}{*}{$0,9-2,03$} & & \\
\hline No (ref) & $142(59,1)$ & $98(40,8)$ & 1,0 & & & \\
\hline \multicolumn{7}{|c|}{ Experiencia previa traumática } \\
\hline Sí & $89(76)$ & $28(23,9)$ & $2,2^{* * *}$ & \multirow{2}{*}{$1,4-3,6$} & & \\
\hline No (ref) & $200(58,3)$ & $143(41,6)$ & 1,0 & & & \\
\hline \multicolumn{7}{|l|}{ Influencia negativa } \\
\hline Sí & $166(67,7)$ & $79(32,2)$ & $1,5^{\star *}$ & \multirow{2}{*}{$1,07-2,20$} & & \\
\hline No (ref) & $123(57,2)$ & $92(42,7)$ & 1,0 & & & \\
\hline \multicolumn{7}{|l|}{ Víctima de equivocación } \\
\hline Sí & $53(62,3)$ & $32(37,6)$ & 0,9 & \multirow{2}{*}{$0,5-1,5$} & & \\
\hline No (ref) & $236(63)$ & $139(37)$ & 1,0 & & & \\
\hline \multicolumn{7}{|l|}{ Percepción salud oral } \\
\hline Buena & $163(58,8)$ & $114(41,1)$ & $1,5^{\star * \star *}$ & \multirow{2}{*}{$1,04-2,2$} & & \\
\hline Mala (ref) & $126(68,8)$ & $57(31,1)$ & 1,0 & & & \\
\hline \multicolumn{7}{|l|}{ Porcentaje de placa } \\
\hline $21-100 \%$ & $277(66,6)$ & $165(37,3)$ & 0,7 & 0432 & & \\
\hline $0-20 \%$ & $12(66,6)$ & $61(33,3)$ & 1,0 & $0,4-3,2$ & & \\
\hline Gingivitis & & & & & & \\
\hline Presencia & $269(58,4)$ & $160(34,7)$ & 0,92 & 07 & & \\
\hline Ausencia (ref) & $20(4,3)$ & $11(2,3)$ & 1,0 & $0,43-1,97$ & & \\
\hline Periodontitis & & & & & & \\
\hline Presencia & $123(62,7)$ & $74(37,5)$ & 0,9 & & & \\
\hline Ausencia (ref) & $166(63,1)$ & $97(36,8)$ & 1,0 & $0,6-1,4$ & & \\
\hline Uso de prótesis & & & & & & \\
\hline Sí & $97(64,2)$ & $54(35,7)$ & 1,09 & & & \\
\hline No (ref) & $192(62,1)$ & $117(37,8)$ & 1,0 & $0,1-1,6$ & & \\
\hline Miedo dental (DFS) & & & & & & \\
\hline Con miedo & $280(78,8)$ & $75(21,1)$ & $39,8^{*}$ & & & \\
\hline Sin miedo (ref) & $9(8,5)$ & $96(91,4)$ & 1,0 & $19,2-82,5$ & $37,8^{x}$ & $17,9-79,5$ \\
\hline $\begin{array}{l}\text { Trastorno de ans } \\
\text { (Goldberg) }\end{array}$ & & & & & & \\
\hline Sí & $124(68,1)$ & $58(31,8)$ & $1,4^{+}$ & 0 & & \\
\hline No (ref) & $165(59,3)$ & $113(40,6)$ & 1,0 & $0,9-\angle, 1$ & & \\
\hline
\end{tabular}

Tabla 4. Asociación entre la ansiedad en la consulta odontológica con variables socio-demográficas, experiencia previa, percepción y salud oral de individuos atendidos en las clínicas de la Facultad de Odontología de la Universidad de Cartagena (Colombia).

Significación del modelo OR $\left(p=0,000 ; \chi^{2}=184,3\right)$. ${ }^{*} p=0,000{ }^{* *} p=0,02{ }^{* * *} p=0,001{ }^{* * * *} p=0,03{ }^{\dagger} p=0,05$ 
igual forma, Field ${ }^{26}$ concluyó que el individuo puede presentar un contagio de las respuestas de miedo.

Debido al instrumento utilizado en la recolección de datos para la determinación del miedo y la ansiedad en la consulta odontológica, este estudio no permite hacer un diagnóstico definitivo, ya que emplea una prueba de cribado y no una prueba diagnóstica; sin embargo, permite hacer una aproximación a la problemática del miedo y la ansiedad dental en la población. Para controlar el sesgo de medición, se incorporaron los instrumentos psicométricos SDAI, DFS y GOLDBERG, que presentan una buena sensibilidad y especificidad y han sido previamente validados en varios países latinoamericanos incluida Colombia ${ }^{1}$.

Tras los resultados obtenidos en la población que asiste a la Facultad de Odontología de la Universidad de Cartagena (Colombia), se puede concluir que el miedo y la ansiedad dental son de origen multifactorial, pudiendo ser inherentes al individuo (como el sexo) o de otra naturaleza psicosocial (como la influencia negativa proveniente la familia o los antecedentes de experiencia traumáticas en la consulta odontológica). Es importante abordar en la consulta odontológica todas las dimensiones del ser humano que puedan influir en el éxito de los tratamientos, como la cultura, sus experiencias previas desde la niñez, o las prácticas de crianza con relación a la atención en salud bucal, con el fin de evitar la aparición y el aumento sintomatológico del miedo y la ansiedad en la consulta.

\section{BIBLIOGRAFÍA}

1. Caycedo C, Cortés OF, Gama R, Rodríguez $\mathrm{H}$, Colorado P, Caycedo $\mathrm{M}$ et al. Ansiedad al tratamiento odontológico: características y diferencias de género. Suma Psicológica. 2008; 15 (1): 259-78.

2. Skaret $E$, Kvale G, Raadal M. General self-efficacy, dental anxiety and multiple fears among 20 -year-olds in Norway. Scand J Psychol. 2003; 44 (4): 331-7.

3. Márquez JA, Navarro MC, Cruz D, Gil J. ¿Por qué se le tiene miedo al dentista?: Estudio descriptivo de la posición de los pacientes de la Sanidad Pública en relación a diferentes factores subyacentes a los miedos dentales. Rev Actual Odontoestomatol Esp. 2004; 9 (2): 165-4.

4. Tickle M, Milsom K, Crawford FI, Aggarwal VR. Predictors of pain associated with routine procedures performed in general dental practice. Community Dent Oral Epidemiol. 2012; 40 (4): 343-50.

5. Hmud R, Walsh L. Ansiedad dental: causas, complicaciones y métodos de manejo. JMID. 2009; 2 (1): 237-48.

6. Armfield JM. Are people with dental fear underrepresented in oral epidemiological surveys? Epidemiol
Psychiatr Sci. 2009; 44 (6): 495-500

7. Álvarez M, Casanova Y. Miedo, ansiedad y fobia al tratamiento estomatológico. Humanidades Médicas. 2006; 6 (1): 1-21.

8. Woodmansey KF. The prevalence of dental anxiety in patients of a University Dental Clinic. J Appl Clin Med Phys. 2005; 54 (1): 59-61.

9. Boman U, Lundgren J, Elfstrom M, Berggren U. Common use of a Fear Survey Schedule for assessment of dental fear among children and adults. Int J Paediatr Dent. 2008; 18 (1): 70-6.

10. Oosterink F, De Jongh A, Hoogstraten J. Prevalence of dental fear and phobia relative to other fear and phobia subtypes. Eur J Oral Sci. 2009; 117 (2): 135-43.

11. López-Soto $O$, Cerezo-Correa $M$, Paz-Delgado $A$. Variables relacionadas con la satisfacción del paciente de los servicios odontológicos. Gerencia y Políticas de Salud. 2010; 9 (18): 124-36.

12. Goldberg $D$, Bridges $K$, Duncan-Jones $P$, Grayson $D$. Detecting anxiety and depression in general medical settings. Br Med J. 1988; 297 (6653): 897-9.

13. Montón C, Pérez-Echevarría MJ, Campos R, García Campayo J, Lobo A. Escalas de ansiedad y depresión de Goldberg: una guía de entrevista eficaz para la detección del malestar psíquico. Aten Prim. 1993; 12 (6): 345-9.

14. Kleinknecht $R$, Klepac $R$, Alexander $L$. Origins and characteristics of fear of dentistry. J Am Dent Assoc. 1973; 86 (4): 842-8.

15. Savithri NV, Rashidah E. Dental fear and utilisation behaviour among antenatal mothers in Seremban District. Ann Dent. 2008; 15 (1): 27-32.

16. Aartman $\mathrm{IH}$. Reliability and validity of the short version of the Dental Anxiety Inventory. Community Dent Oral Epidemiol. 1998; 26 (5): 350-4.

17. Klein $\mathrm{H}$, Palmer $\mathrm{C}$. Studies on dental caries. Dental status and dental need of elementals schools children. Pub Health Rep. 1938; 53 (38): 1685-90.

18. Feitosa $R$, de carvalho $P$, de Luna $G$, de Souza A, Pereira $J$, da Silva $M$ et al. Ansiedade frente ao tratamento odontológico: prevalência e fatores predictores em brasileiros. Cien Saude Colet. 2012; 17 (7): 1915-22.

19. Ferreira CM, Gurgel-Filho ED, Bönecker-Valverde G, Moura EH, de Deus G, Coutinho-Filho T. Ansiedade odontológica: nível, prevalência e comportamento. Revista Brasileira em Promoção da Saúde. 2012; 17 (2): 51-5.

20. Pardo G, Sandoval A, Umbarila D. Adolescencia y Depresión. Rev Colombiana de Psicologia. 2003; XXXII (4): 341-56.

21. Arenas MC, Puigcerver A. Diferencias entre hombres $y$ mujeres en los trastornos de ansiedad: una aproximación psicobiológica. Escritos de Psicología. 2009; 3 (1): 20-9.

22. Ritsner M, Ponizovsky A, Nechamkin Y, Modai I. Gender differences in psychosocial risk factors for psychological distress among immigrants. Compr Psychiatry. 2001; 42 (2): $151-60$

23. Edmunds $\mathrm{R}$, Buchanan $\mathrm{H}$. Cognitive vulnerability and the aetiology and maintenance of dental anxiety. Community Dent Oral Epidemiol. 2012; 40 (1): 17-25.

24. Oliveira $M$, Colares V. The relationship between dental anxiety and dental pain in children aged 18 to 59 months: a study in Recife, Pernambuco State, Brazil. Cad Saude Publica. 2009; 25 (4): 743-50.

25. Carrillo J, Pernia I, Alvarez C, García T. Odontología de baja agresividad: esto ya es el presente. Gaceta dental: Industria y profesiones. 2005; 1 (162): 106-26.

26. Field A, Lawson J. The verbal information pathway to fear and subsequent causal learning in children. Cogn Emot. 2008; 22 (3): 459-79. 